\title{
Molecular targeting of vulnerable RNA sequences in SARS CoV-2: identifying clinical feasibility
}

\author{
G. Ian Gallicano $\mathbb{1}^{1} \cdot$ John L. Casey ${ }^{2} \cdot$ Jiayu Fu$^{1} \cdot$ Samiksha Mahapatra ${ }^{1}$
}

Received: 14 August 2020 / Revised: 8 October 2020 / Accepted: 27 October 2020 / Published online: 12 November 2020

(c) Springer Nature Limited 2020

\begin{abstract}
Covid-19 (SARS CoV-2) has become a deadly, world-wide pandemic. Although most who are infected survive, complications from the virus can be pronounced and long-lasting. To date, of all the respiratory viruses including influenza and coronaviruses, only influenza has had a drug (i.e., Tamiflu) specifically targeted to treat and prevent infection. As a result, additional agents that specifically target viral production and are clinically feasible are needed to alleviate respiratory viral infections. The idea of using a miRNA/siRNA molecular approach for treating various diseases was postulated over a decade ago; however, only within the past few years has it become feasible. One technological advancement has been the molecular linkage of lipophilic moieties to mi/siRNAs in order to bypass the need for enveloping these inhibitory RNAs in lipid-based transfection reagents, which could irritate the airway if inhaled. Here we show that siRNAs and miRNAs inhibit SARS CoV-2 spike protein production in a dose-dependent manner in both HEK293 cells and a primary human airway tracheal cell line. We also show that this inhibition is equally robust using a clinically relevant siRNA that does not need to be prepped with a transfection reagent.
\end{abstract}

\section{Introduction}

Common respiratory viruses such as rhinovirus, respiratory syncytial virus, human metapneumovirus, influenza virus (IV), and coronavirus (hCoV) have been studied for decades [1]. In general, most who are infected survive; however, the death rate from complications (e.g., viral pneumonia) for many of these viruses remains high. The arsenal of agents for battling these types of viruses ranges from potent vaccines to over-the-counter medications for alleviating symptoms. With the exception of Tamiflu for IV, few agents exist, either pharmacological or molecular, that are able to directly attack the replication, construction, or infectious ability of these viruses. This problem is exacerbated by the fact that viral immunity, particularly mucosal,

$\triangle$ G. Ian Gallicano

gig@georgetown.edu

1 Department of Biochemistry and Molecular and Cellular Biology, Georgetown University Medical Center, Washington, DC 20057, USA

2 Department of Microbiology, Georgetown University Medical Center, 3900 Reservoir Rd. NW, Washington, DC 20057, USA appears to wane over just a few years. Interestingly, mutation does not seem to be prevalent within the coronaviruses that are widely circulating. As a result, SARS $\mathrm{CoV}-2$ viral ssRNA coding regions are vulnerable to genetic targeting, an approach that would result in translational suppression of proteins necessary for virus production/infection. A meticulous description of SARS CoV-2 can be found in ref. [2], which elucidates the mechanistic complexities that regulate the SARS CoV-2 cycle of infection. Based on existing information for SARS CoV-2, we have devised a strategy to stop, or at least slow, the production of infectious virions. This strategy is loosely based on ongoing work in which we employed a paradigm shift for treating heart failure using a molecular, genetargeting approach [3]. Specifically, we use miRNA technology to target genes known to cause heart failure in order to return cardiac function to normal parameters. Given previous success, we tested whether this molecular approach could be effective against SARS CoV-2 and hCoVs in general.

The hypothesis is that synthetic miRNAs (or alternatively siRNAs) can be used to target and suppress specific translation events critical for SARS CoV-2 production and/ or infection. MiRNAs are small noncoding RNA molecules (containing about 22 nucleotides) found in plants, animals, 
and some viruses. In vertebrates, miRNAs are predicted to regulate timing of protein expression, epigenetic gene regulation, and overall protein homeostasis within cells [4-9]. While miRNAs generally function by targeting the $3^{\prime}$ UTR of an mRNA through their seed sequence consisting of 7-8 bases (although some can target $5^{\prime}$ UTR and open reading frame (ORF)), siRNAs, which are artificially synthesized are generally perfect matches to the ORF of mRNAs. When either miRNA or siRNA is used to target an mRNA, posttranscriptional silencing of gene expression is the direct result. All hCoVs must use translational machinery to generate the proteins necessary for replication and infection. Consequently, translation of these proteins is susceptible to targeted suppression by miRNAs and/or siRNAs.

The idea of using a miRNA/siRNA molecular approach for treating various diseases was postulated over a decade ago; however, only within the past few years has it become feasible. A prime example illustrating the clinical implementation of this novel paradigm is the recent (2017) antisense agent IONIS-HTTRx (Ionis Inc.) for treating Huntington's disease [HTT] [10]. This therapeutic approach is showing great promise not only with respect to safety but also clearing away all mutant forms of HTT protein in phase 1 and 2 clinical trials. Antisense technologies are now becoming feasible for treating cancers [11], muscular dystrophy [10], and some viral infections [12-14]. Based on over a decade of experience using siRNA, miRNA mimics, and inhibitors [3, 15-17,], we are proposing to use this technology here for suppressing SARS CoV-2 infection/ production. The entire SARS CoV-2 sequence was released early in 2020. This information has significantly sped up our ability to test our hypothesis of targeting SARS CoV-2 RNA components.

\section{Results and discussion}

Two cell types, HEK293 cells and primary human tracheal cells (hpTCs) were employed to test the hypothesis that siRNAs or miRNAs could suppress SARS CoV-2 spike expression. Using immunofluorescence analyses of both HEK293 cells and hpTCs, spike protein expression was clearly evident within $48 \mathrm{~h}$ of transfection (HEK293 cells) or electroporation (hpTCs) of spike protein expression construct (Fig. 1A-D). To alleviate concern that siRNAs or miRNAs could consistently enter cells, a mimic control conjugated to Dy547 was transfected at various concentrations. $200 \mathrm{nM}$ provided good evidence that small RNAs could easily move into both types of cells (Fig. 1E, F).

HEK293 cells were used first to investigate the ability of each siRNA to suppress production of spike protein. We found that siRNA1 and 2 alone or in combination could suppress spike production in a dose-dependent manner
(Fig. 2A, B). Quantification (Fig. 2B) of at least three Western blots identified a significant difference in spike protein expression primarily at the 200 and $20 \mathrm{nM}$ concentrations of siRNAs. The $2 \mathrm{nM}$ concentration was not able to significantly block spike protein translation. Delving further into the mechanism of suppression, semiquantitative rt-PCR revealed that siRNA suppression is caused by degradation of spike mRNA (Fig. 2C, D). Concomitantly with protein expression, quantification of three semiquantitative RT-PCRs (Fig. 2D) revealed a significant decrease in spike mRNA primarily at the 200-nM siRNA concentration. These data provide evidence that siRNAs lead to degradation of spike mRNA.

We duplicated these experiments using two different miRNAs, miR510-3p and miR624-5p, both of which were predicted using miRDB.org to target the ORF of the viral spike RNA. Generally, miRNAs target sequences within 3'UTRs of mRNAs; however, we surmised that miRNA mimics might fare better as an antiviral agent because they are more easily metabolized by nucleases within cells and, thus, any off-target events would be minimized. We found that miRNA-624-5p was more effective than miRNA 5103p (Fig. 2E). Quantification of at least three Western blots revealed miR624-5p significantly suppressed translation of spike mRNA in a dose-dependent manner (Fig. 2F). Interestingly, while the semiquantitative RTPCR revealed that siRNA degraded spike mRNA (Fig. 2D), miRNA functioned differently as spike mRNA did not seem to be degraded (Fig. 2G). Significant difference was measured only at the 200-nM dosage for miR510-3p (Fig. 2H).

The next step was to identify if siRNA $1+2$ and miRNA $1+2$ could suppress spike production in hpTCs. While transfection of SARS CoV-2 spike plasmid using Mirius TransIT-X2 ${ }^{\circ}$ Transfection Reagent worked well with HEK293 cells, hpTCs were difficult to transfect. As a result, electroporation was used to introduce spike cDNA into hpTCs. Twenty four hours after electroporation of spike plasmid, $200 \mathrm{nM}$ of each small RNA (the concentration seen to virtually completely suppress spike in HEK293 cells) was transfected resulting in marked reduction in spike protein expression in both siRNA $1+2$ and miRNA1 +2 samples (Fig. 3A). These results provided clear evidence that specific small RNAs could be used as a distinct strategy for suppressing functional aspects of respiratory viruses such as SARS CoV-2 in human airway tracheal cells.

siRNA1 was then modified with a cholesterol moiety and then introduced, without transfection reagent, into SARS CoV-2 spike expressing cells. The idea here was to identify a more clinically feasible approach for delivering an antisense RNA to suppress spike protein production. The data in Fig. 3B, C reveal that siRNA1/NT (non-transfection reagent) suppressed spike protein production in a 
Fig. 1 SARS CoV-2 spike protein is expressed in both HEK293 cells and hpTCs. A Transfection of the CMVSARS CoV-2-Flag plasmid results in good expression within $48 \mathrm{~h}$. Arrowheads point to spikepositive cells. B Transmitted light image of cells in $\mathbf{A}$ showing that cells that were $75 \%$ confluent when transfected, were $90 \%$ confluent $48 \mathrm{~h}$ later. hpTCs that were un-electroporated (C) showed no cells positive for spike protein, while hpTCs electroporated with SARS CoV2 spike plasmid (D) show spike protein production (arrowheads) within $48 \mathrm{~h}$. Insets show transmitted light images of cells. E, F A mimic, 21 base small RNA linked to a fluorescent Dy547 dye shows that both cell types easily internalize si/ miRNAs. $E=20 \times, F=40 \times$
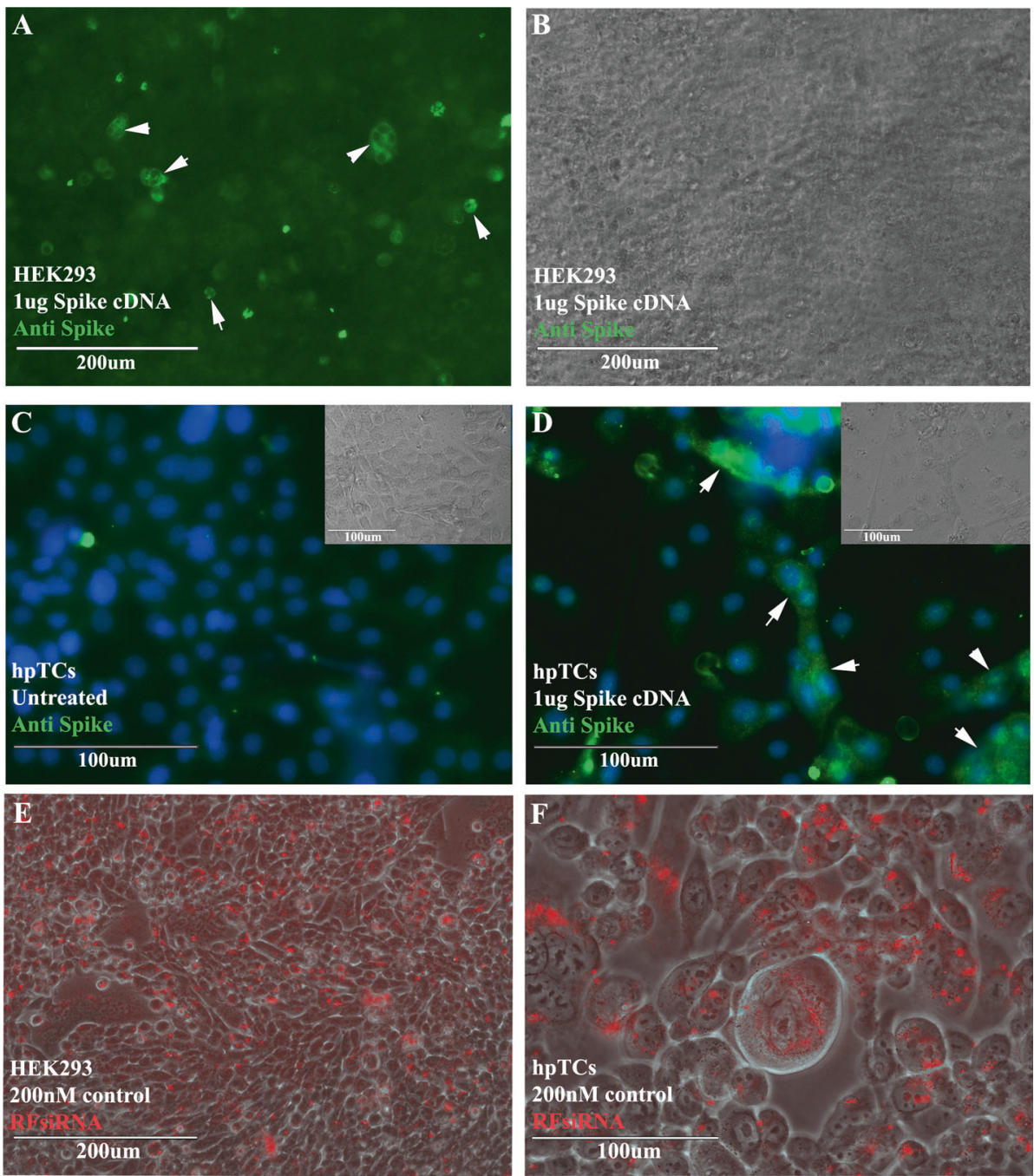

dose-dependent manner in HEK293 cells (Fig. 3B). Using siRNA1/NT in hpTCs, it was evident that this single siRNA could suppress spike protein expression in a dose-dependent manner without the need of a potentially irritating lipidbased transfection reagent.

Finally, off-targets and cell proliferation/viability were analyzed to alleviate concerns of siRNA/miRNA inadvertent effects. Cell cycle/growth was analyzed by comparing untreated cells to cells treated with siRNA and miRNA1 +2 . Ki67 staining and quantification clearly showed no significant difference between si/miRNA-treated and -untreated hpTCS (Fig. 4A-D). Measuring cell growth over $72 \mathrm{~h}$ using a Biorad TC20 cell counter also showed no significant differences between untreated and si/miRNAtreated hpTCs (Fig. 4E). Cell viability quantification also resulted in no significant difference between untreated and si/miRNA-treated hpTCs (Fig. 4F). We surmise from these data that introduction of clinically relevant siRNA1/NT would not induce abnormal cell growth or abnormal cell death.
Off-targets are always a concern when introducing RNAi into cells. Both siRNAs were blasted in miRDB.org to identify if there were perfect matches against any known genes in humans. We found no genes in the human genome that were perfect matches for siRNA1 or 2 . Two genes, however, retinoblastoma-1 and MADS box transcription enhancer factor 2, polypeptide $\mathrm{C}$ (Mef2C) were $75 \%$ matches (16/21 bases). As a result, we tested if Mef2C could be affected by either siRNA. Figure $4 \mathrm{G}-\mathrm{L}$ showed that Mef2C expression was not affected by siRNA1 or 2.

With respect to miRNAs, there were 25 genes with a "target score" of 90/100 (90\%) or higher. Target scores are defined by a variety of algorithms that predict if that miRNA has the ability to bind to an mRNA. Testing if any of these 25 genes can be downregulated by miRNA1 or 2 will need to be investigated before they can be tested in a clinical setting.

Taken together, these data provide strong evidence that siRNA and possibly miRNAs could prevent the production 

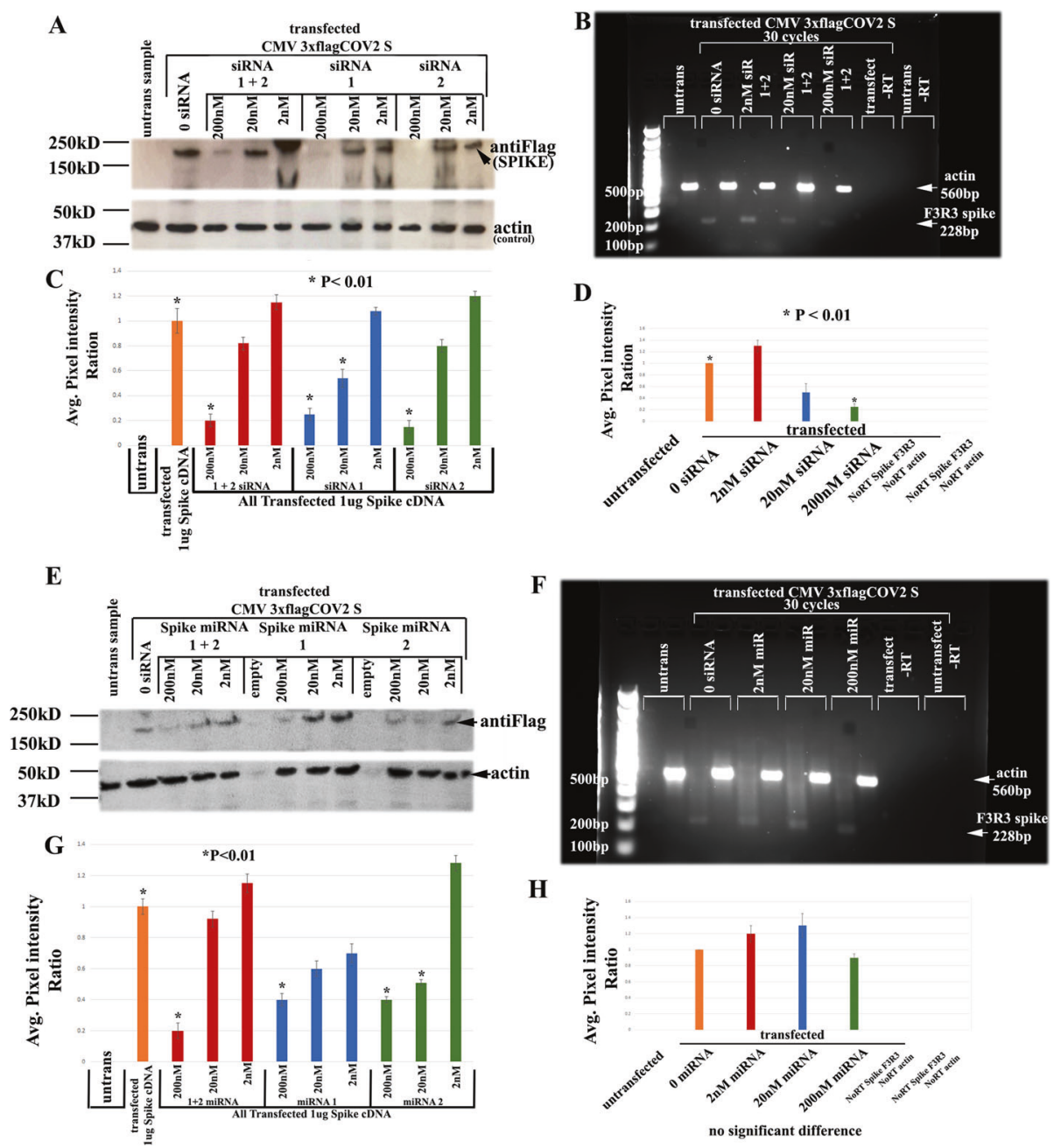

$\mathbf{H}$
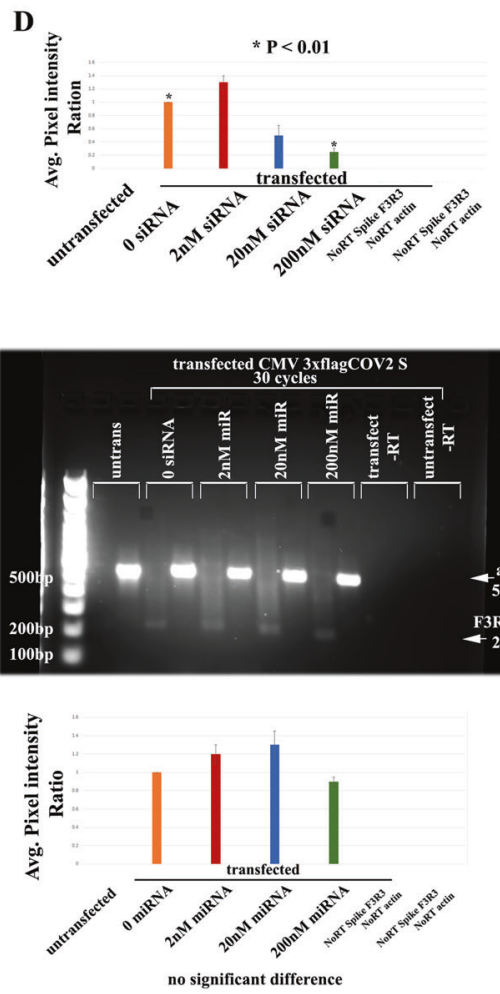

Fig. 2 Experiments analyzing si and miRNA effects on spike protein and mRNA production. A Western blot showing dosedependent effects of siRNA on viral spike protein production. Both spike siRNAs target and prevent translation. B Intensity of bands were quantified and graphed $(N=3)$. Asterisks represent significant differences between no spike siRNA (0 drug) and all 200-nM spike siRNAs. C Semiquantitative rt-PCR reveals a mechanism for the loss in protein production in the Western. Higher levels of spike siRNA result in lower levels of spike RNA. D Intensity of bands at increasing siRNA dosage was quantified and graphed $(N=4)$. Asterisks represent significant differences between no spike siRNA and 200-nM siRNAs.

of an important infectious component (i.e., spike) for SARS CoV-2. We are aware that other viral particles might still be generated with this approach; however, even if an entire virus is generated and assembled properly, once exuded, it would be incapable of infecting neighboring cells without spike protein production.

We are currently working on loading siRNA/NT and miRNAs into a Microsprayer Aerosolizer (Penn-Century Inc.) in order to spray the siRNA/NT or miRNA complexes into cells. We are also in the process of identifying if this technology suppresses other vulnerable Covid sequences such as the nucleocapsid protein or specific membrane

E-H Similar experiments were performed to analyze miRNA effects on spike expression. Both miRNAs were observed to suppress spike expression; however, miRNA624 seem to be more effective in a dosedependent manner. F Quantification of Western blots identified miRNA624-5p (miRNA2) slightly more effective than miRNA 510-3p (miRNA1). G Semiquantitative RTPCR reveals a mechanism for the loss in protein production in the Western. Spike mRNA remained intact at all concentrations of miRNA suggesting that miRNAs 1 and 2 suppress spike protein expression by suppressing translation and not mRNA degradation. $\mathbf{H}$ Intensity of bands at increasing siRNA dosage was quantified and graphed $(N=4)$.

proteins. We are confident that this technology can be quickly moved from bench to bedside.

\section{Methods}

\section{Cell culture}

HEK293 cells were obtained from the tissue and cell core at Lombardi Comprehensive Cancer Center and cultured in high glucose DMEM with Penicillin/Streptomycin. hpTCs (ScienCell Cat\# 3220) were grown in Bronchial epithelial 


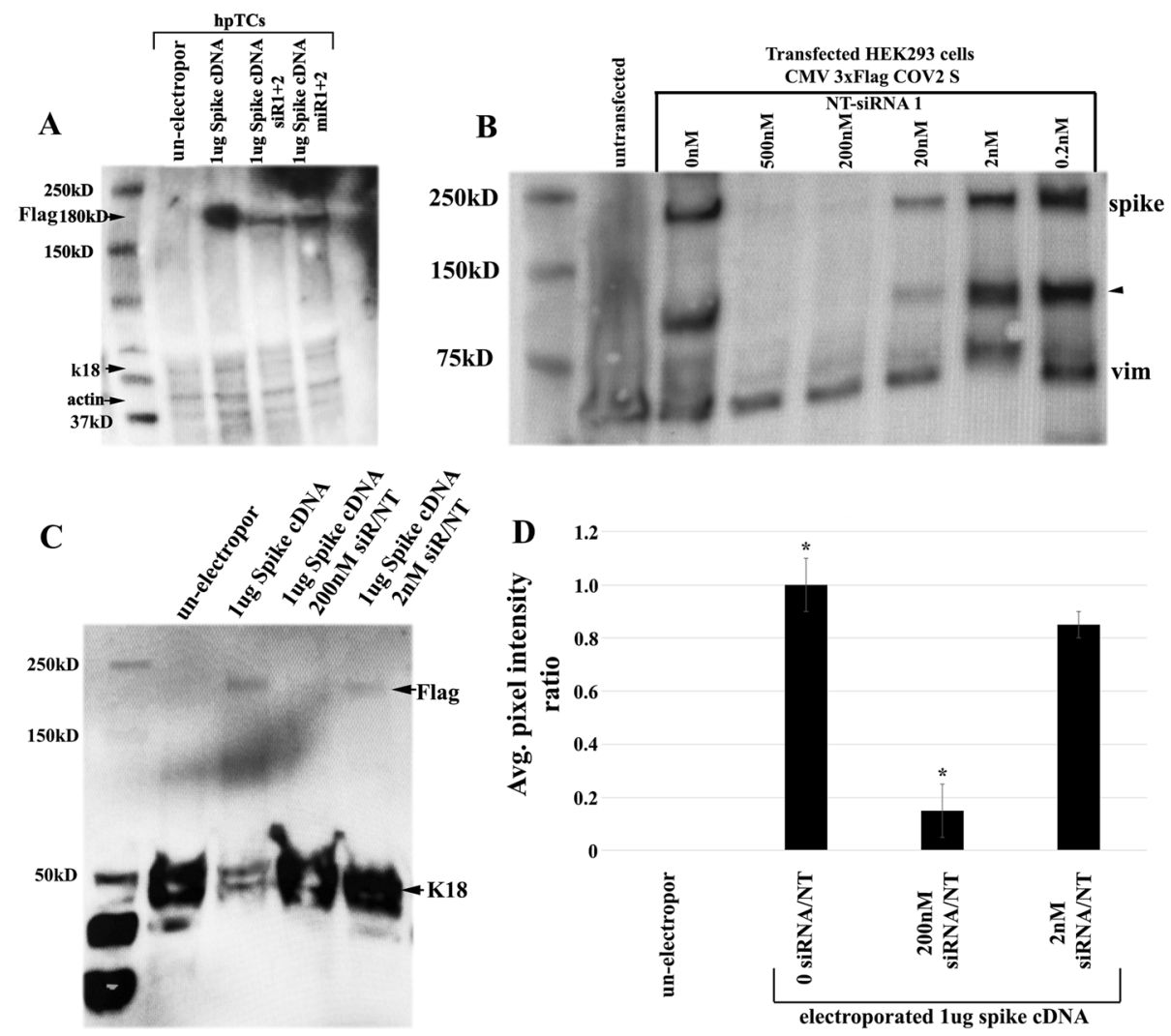

Fig. 3 Identifying siRNA, miRNA, and siRNA/NT efficacies. A htPCs electroporated with SARS CoV-2 spike plasmid (lane 3) showed marked suppression of spike protein when transfected with 200-nM siRNAs (Lane 4) or miRNAs (Lane 5). Flag antibody was used in this experiment to identify spike protein. Antibodies to K18 and actin were used for loading controls. B siRNA/NT introduction (without transfection reagent) into HEK293 cells co-transfected with SARS CoV-2 spike plasmid showed a suppression of spike protein in a dose-dependent manner. Spike antibody was used in this experiment to

cell medium along with bronchial epithelial cell growth supplement (ScienCell Cat\#3211). Cells were counted using a BioRad inc. TC20 cell counter. Cells were transfected with TransIT-X2 ${ }^{\circ}$ Transfection Reagent (Mirius Bio Cat\# MIR6003).

\section{Semiquantitative rt-PCR}

Semiquantitative rt-PCR was used to identify the mechanism of suppression for Covid-19 spike mRNA translation 2 days after transfection during exponential phase at 2, 20, and 200$\mathrm{nM}$ dilutions of siRNA $1+2$ or miRNA $1+2$. Total RNA was isolated using the miRNeasy Mini Kit (Qiagen, Cat \#217004). Concentrations of the extracted RNA were determined using NanoDrop 2000. Ten microliters of total RNA were treated with RQ1 RNase Free DNase (Promega; cat. No. M6106) to degrade all genomic and spike plasmid. cDNA was prepared using the High Capacity cDNA Reverse Transcription Kit (AppliedBiosystems; cat. No. 4368814). identify spike protein. A lower band is a potential proteolytic product often detected with this antibody (arrowhead). Vimentin was used as loading control. C siRNA/NT tested in htPCs electroporated with SARS CoV-2 spike plasmid showed a dose-dependent suppression of spike protein. D Quantification of four Western blot experiments showed significant difference between $200 \mathrm{nM}$ and electroporated htPCs. *significant difference $p<0.05$. Virtually no spike protein was detected with 200-nM siRNA/NT.

Semiquantitative PCR was performed using MyTaq Red Mix (Bioline; cat. No. BIO-25043) at 25, 30, and 35 cycles. Using cDNA generated from actin mRNA as an internal control, we verified that 30 cycles of PCR were in the log phase of amplification for all treatments. Two different sets of reverse primers were used to generate cDNA from spike mRNA. The same two primers along with their forward counterparts were employed to amplify cDNA. Primers used were: Actin, up: CTCTTCCAGCCTTCCTTCC; low: TCGGCCACATTGTG AACTTT. Spike F2R2 315 b.p., up: TGCACAAGCTTTA AACACGCT; low: ACACCATGAGGTGCTGACTG; spike F3R3 228 b.p., up: CGTCTTGACAAAGTTGAGGCTG; low: ATGAGGTGCTGACTGAGGGA.

\section{Western blot}

293 and Tracheal cells were trypsinized with $0.25 \%$ TrypsinEDTA and then centrifuged at $1000 \mathrm{~g}$ for $5 \mathrm{~min}$ before addition of sample buffer. The whole cell lysates were then 
Fig. 4 The effects of siRNAs and miRNAs on cell cycle, viability, and off-targets were analyzed in hpTCs. Antibodies to Ki67 revealed nuclear staining (green) in untreated (A), 200-nM siRNA treated $(\mathbf{B})$, and 200-nM miRNA (C). D

Quantification of hundreds of cells from four different experiments showed no significant difference between untreated and treated hpTCs. E Cell proliferation also was not effected by si/miRNAs. F Cell viability measured by trypan blue revealed that $\mathrm{si} / \mathrm{miRNAs}$ did not cause cell death. G-J Mef2C was listed as a predicted target (75\% match) of siRNA1. Antibodies directed against Mef2C revealed staining in both untreated (G-H) and siRNAtreated (I-J) hpTCs. K Nuclei positive for Mef2C were counted and graphed. No significant difference was found $(N=4)$. L Western analyses further confirmed no difference in protein expression between untreated and siRNA-treated cells. This Mef2C antibody often detects Mef2c protein as a doublet. Actin serves as the loading control.
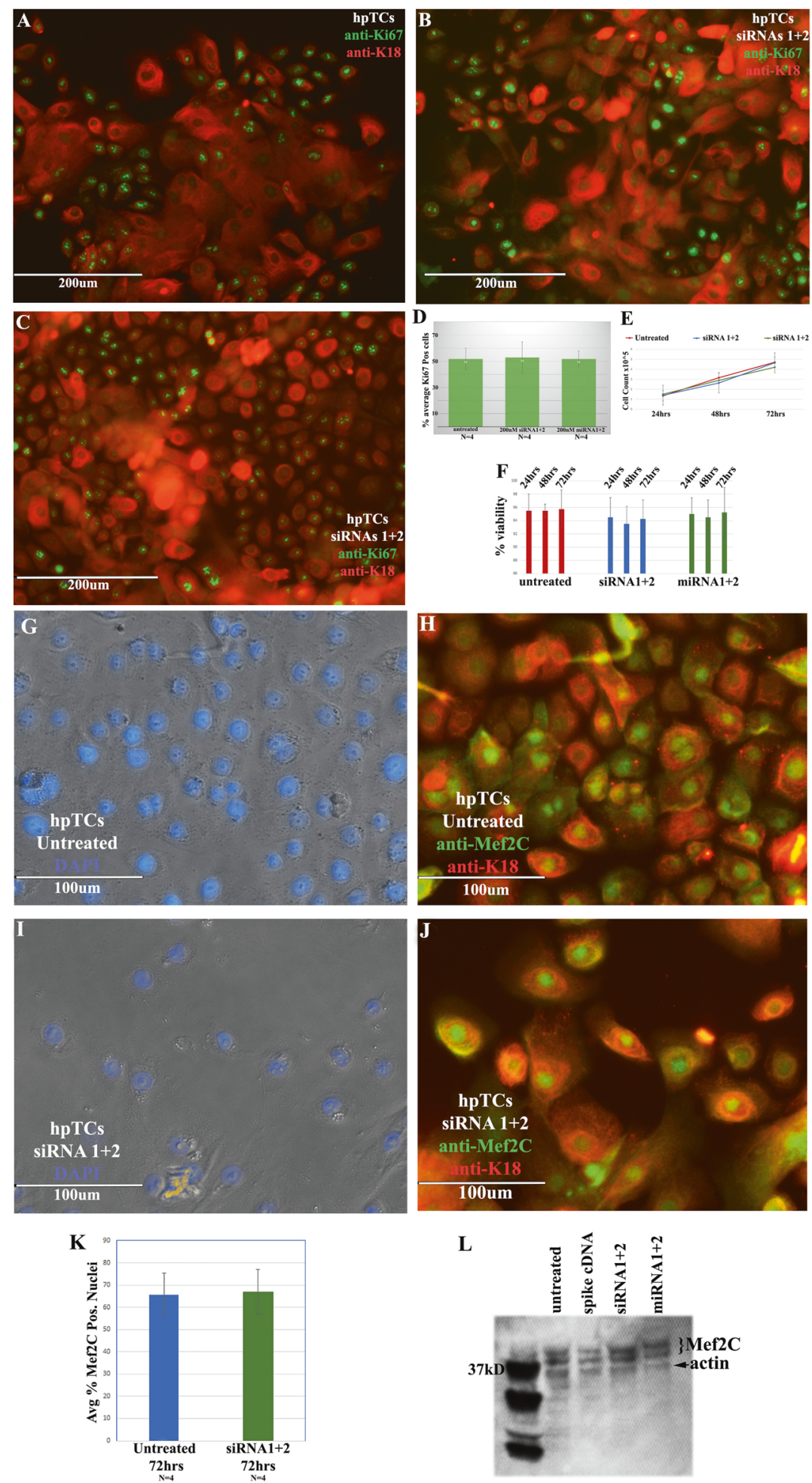

heated on for $10 \mathrm{~min}$ at $95^{\circ}$. Lysates were loaded on $4-20 \%$ gels (Biorad) and ran at $250 \mathrm{~V}$. Consequently, the gel was transferred to a $0.22-\mu \mathrm{M}$ PVDF membrane (Biorad) using the Trans-blot Turbo RTA PVDF Midi Transfer kit (Biorad). Blots were blocked in 2\% nonfat dry milk/TBST (pH 7.5) and washed with $1 \mathrm{X}$ TBST/1\% Tween. Western blot antibodies recognized spike (Genetex Cat \#GTX632604), Rb1 (Proteintech Cat \#10048-2-Ig), Mef2C (Proteintech Cat \#10056-1AP), Flag (Abcam Cat \#ab205606), Keratin 18 (EMD Millipore Cat\#) Secondary antibodies were donkey anti mouse (Jackson Immunoresearch), goat anti rabbit (Jackson Immunoresearch) and donkey anti rat (EMD Millipore). 


\section{Immunofluorescence}

293 cells and Tracheal cells were fixed with $4 \%$ paraformaldehyde and stained for Flag (Abcam), spike (Genetex), Mef2C (Proteintech), Rb1 (Proteintech). Control antibodies Vimentin (Sigma Aldrich Cat \#V4630) and Keratin 18 (EMD Millipore) stained 293 and tracheal cells, respectively. Secondary antibodies conjugated with Alexa Fluor (Life Technologies) were used. Stained cells were visualized using an EVOS AMG inverted microscope.

\section{Statistics}

All statistics were performed using the student $t$ test.

\section{Generation of si/miRNA sequences}

siRNAs sequences were generated using the spike cDNA sequence from https://www.ncbi.nlm.nih.gov/nuccore/NC_ 045512.2 ?rom $=21563 \&$ to $=25384 \&$ report $=$ genbank followed by and blasting from the spike sequence at https://www.invivogen.com/sirnawizard/siRNA.php. Two siRNAs were picked from 11 based on different GC\% contents $(47.62 \%$ for siRNA1 and $52.38 \%$ for siRNA2) and fewest base-matches with genes in the human genome. miRNAs were blasted from miRDB.org. The two miRNAs chosen were based on the highest predicted target percentage and the lowest number of off-targets. All transfected siRNAs and miRNAs were purchased from Thermofisher Inc. Modified siRNA requiring no transfection reagent (NT siRNAs) was purchased from Horizon Inspired Cell Solutions Inc. Sars-Cov-2 Spike cDNA (pCMV14-3X-Flag-SARS-CoV-2 S) was purchased from Addgene Inc [18].

siRNA needing transfection reagent for transfection into cells.

siRNA1-Sense AGACCCAGUCCCUACUUAU Antisense-AUAAGUAGGGACUGGGUCUU. siRNA2-Sense CCGUGAUCCACAGACACUU Antisense-AAGUGUCUGUGGAUCACGG.

miRNA1-hsa-miR624-5p.

miRNA2-hsa-miR510-3p.

All were purchased from Life Technologies Inc.

The following siRNA was linked to a lipid moiety and did not need a transfection reagent for entering cells. Purchased from Horizon Discovery/Dharmacon Inc.

siRNA1-SPIKE: length: 21.

Sense: 5'A.G.A.C.C.C.A.G.U.C.C.C.U.A.C.U.U.A.U.U. U $3^{\prime}$.

Antisense: 5'5'-P.A.U.A.A.G.U.A.G.G.G.A.C.U.G.G.G. U.C.U.U.U $3^{\prime}$.
Acknowledgements We need to thank Dr Nady Golestaneh for use of her EVOS AMG inverted microscope and secondary antibody reagents. We also thank Vaughn Gallicano for acquiring immunofluorescence images used for data gathering and publication. We also thank to Olympia Hatzilambrou for assisting in off-target identification. This work was funded by a Covid-19 Pilot Project Award from Georgetown University Medical Center.

\section{Compliance with ethical standards}

Conflict of interest The authors declare that they have no conflict of interest.

Publisher's note Springer Nature remains neutral with regard to jurisdictional claims in published maps and institutional affiliations.

\section{References}

1. Shi T, Arnott A, Semogas I, Falsey AR, Openshaw P, Wedzicha $\mathrm{JA}$, et al. The etiological role of common respiratory viruses in acute respiratory infections in older adults: a systematic review and meta-analysis. J Infect Dis. 2020;222(Supplement_7):S563-9. https://doi.org/10.1093/infdis/jiy662.

2. Fehr AR, Perlman S. Coronaviruses: an overview of their replication and pathogenesis. Methods Mol Biol. 2015;1282:1-23.

3. Zhang Z, Ursin R, Mahapatra S, Gallicano GI. CRISPR/CAS9 ablation of individual miRNAs from a miRNA family reveals their individual efficacies for regulating cardiac differentiation. Mech Dev. 2018;150:10-20.

4. Lal A, Navarro F, Maher CA, Maliszewski LE, Yan N, O'Day E, et al. miR-24 Inhibits cell proliferation by targeting E2F2, MYC, and other cell-cycle genes via binding to "seedless" 3'UTR microRNA recognition elements. Mol Cell. 2009;35: 610-25.

5. Larabee SM, Coia H, Jones S, Cheung E, Gallicano GI. miRNA-17 members that target Bmpr2 influence signaling mechanisms important for embryonic stem cell differentiation in vitro and gastrulation in embryos. Stem Cells Dev. 2015;24:354-71.

6. Ventura A, Young AG, Winslow MM, Lintault L, Meissner A, Erkeland SJ, et al. Targeted deletion reveals essential and overlapping functions of the miR-17 through 92 family of miRNA clusters. Cell. 2008;132:875-86.

7. Wang J, Cao N, Yuan M, Cui H, Tang Y, Qin L, et al. MicroRNA$125 \mathrm{~b} / \mathrm{Lin} 28$ pathway contributes to the mesendodermal fate decision of embryonic stem cells. Stem Cells Dev. 2012;21:1524-37.

8. Wu W, Takanashi M, Borjigin N, Ohno SI, Fujita K, Hoshino S, et al. MicroRNA-18a modulates STAT3 activity through negative regulation of PIAS3 during gastric adenocarcinogenesis. Br J Cancer. 2013;108:653-61.

9. Zhu W, He J, Chen D, Zhang B, Xu L, Ma H, et al. Expression of miR-29c, miR-93, and miR-429 as potential biomarkers for detection of early stage non-small lung cancer. PLoS ONE. 2014;9:e87780. https://doi.org/10.1371/journal.pone. 0087780.

10. Pfister EL, Chase KO, Sun H, Kennington LA, Conroy F, Johnson E, et al. Safe and efficient silencing with a Pol II, but Not a Pol lII, promoter expressing an artificial miRNA targeting human huntingtin. Mol Ther Nucleic Acids. 2017;7:324-34.

11. Rupaimoole R, Slack FJ. MicroRNA therapeutics: towards a new era for the management of cancer and other diseases. Nat Rev Drug Discov. 2017;16:203-22. 
12. Adams BD, Parsons C, Walker L, Zhang WC, Slack FJ. Targeting noncoding RNAs in disease. J Clin Invest. 2017;127: 761-71.

13. Matsui M, Corey DR. Non-coding RNAs as drug targets. Nat Rev Drug Discov. 2017;16:167-79.

14. Ghosh S, Firdous SM, Nath A. siRNA could be a potential therapy for Covid-19. EXCLI J. 2020;19:528-31.

15. Foshay KM, Gallicano GI. Small RNAs, big potential: the role of MicroRNAs in stem cell function. Curr Stem Cell Res Ther. 2007;2:264-71.
16. Foshay KM, Gallicano GI. miR-17 family miRNAs are expressed during early mammalian development and regulate stem cell differentiation. Dev Biol. 2009;326:431-43.

17. Badodi S, Baruffaldi F, Ganassi M, Battini R, Molinari S. Phosphorylation-dependent degradation of MEF2C contributes to regulate G2/M transition. Cell Cycle. 2015;14:1517-28. https:// doi.org/10.1080/15384101.2015.1026519.

18. Ou X, Liu Y, Lei X, Li P, Mi D, Ren L, et al. Characterization of spike glycoprotein of SARS-CoV-2 on virus entry and its immune cross-reactivity with SARS-CoV. Nat Commun. 2020;11. 